\title{
Instability of nuclear chromatin in the ejaculated spermatozoa of fertile men*
}

\author{
W. F. Blazak $\dagger \ddagger$ and J. W. Overstreet $\uparrow \S$ \\ Departments of $\dagger$ Human Anatomy and of §Obstetrics and Gynecology, School of Medicine, \\ University of California, Davis, California 95616, U.S.A.
}

\begin{abstract}
Summary. In the present study of 15 fertile men, a mean of $89 \pm 3 \%$ of ejaculated cells had nuclei which were stable after treatment with $1 \%$ sodium dodecyl sulphate (SDS). Examination of repeated ejaculates demonstrated the constancy of the response of ejaculated spermatozoa from different men to SDS treatment. After 5 cycles of washing, the percentages of cells with stable nuclei after SDS treatment declined from $85 \pm 3$ to $46 \pm 6 \%$ and ranged from 12 to $66 \%$ for different men. The supernatant derived from the first wash inhibited the nuclear chromatin decondensation of washed spermatozoa. Treatment of washed spermatozoa with $1 \mathrm{mm-zinc}$ (which binds to $-\mathrm{SH}$ groups) or $1 \mathrm{mM}$-copper (which promotes oxidation of $-\mathrm{SH}$ groups to $-\mathrm{S}-\mathrm{S}-$ ) resulted in a significant increase in the proportion of cells with stable nuclear chromatin. However, the stabilizing effects of zinc could be reversed with continued washing of the cells, whereas the nuclei of copper-treated cells remained stable to SDS after additional washing. We conclude that (1) the number of spermatozoa with nuclei that are insufficiently stabilized by disulphide bonds is much higher in the semen of fertile men than was previously thought, (2) significant differences exist among fertile men in the proportions of ejaculated spermatozoa extensively stabilized by disulphide bonds, and (3) removal of seminal plasma and/or cellular contaminants (zinc) is necessary to unveil the instability of the nuclear chromatin in the ejaculated spermatozoa of fertile men.
\end{abstract}

\section{Introduction}

Human spermatozoa, like those of other mammals, are not fully mature when released from the seminiferous epithelium and must complete maturation during transit through the epididymis. The changes in human sperm function which take place within the epididymis include development of the capacity for progressive motility (Belonoschkin, 1942; Bedford, Calvin \& Cooper, 1973b), and perhaps the capacity to fertilize the ovum (Hinrichsen \& Blaquier, 1980). The changes within the sperm cell which underlie the development of these functions include alterations of the sperm surface and structural reinforcement of the sperm midpiece and tail which results from the formation of disulphide bonds (Bedford et al., 1973b). The simultaneous formation of disulphide bonds within nuclear protamines results in an increase in the stability of the sperm nucleus during epididymal passage (Bedford et al., 1973b; Saowaros \& Panyim, 1979). Thus, human spermatozoa appear to require epididymal maturation in order to achieve fertility as do the sperm cells of laboratory animals (Orgebin-Crist, 1969; Bedford, 1975).

* Reprint requests to Dr J. W. Overstreet.

† Present address: Genetic Toxicology, SRI International, 33 Ravenswood Avenue, Menlo Park, California, 94025, U.S.A. 
In apparent contrast to other mammals, however, the transit time of spermatozoa through the human epididymis is very short (Amann \& Howards, 1980), and highly variable among individuals (Rowley, Teshima \& Heller, 1970). It is not surprising, therefore, that the human ejaculate contains spermatozoa with various degrees of nuclear stability (Bedford, Bent \& Calvin, 1973a). When ejaculated human spermatozoa were exposed to a detergent, sodium dodecyl sulphate (SDS), alone or in combination with the disulphide bond-cleaving agent dithiothreitol (DTT) (Bedford et al., 1973a), significant heterogeneity was observed amongst spermatozoa in the same ejaculate as well as between ejaculates from different semen donors in the degree of nuclear chromatin decondensation (Bedford et al., 1973a; Kvist \& Eliasson, 1980). Irregularities in the nuclear chromatin condensation of bull spermatozoa have been associated with infertility (McCosker, 1969; Evenson, Darzynkiewicz \& Melamed, 1980), and it has been suggested that there may be a relationship between human male infertility or embryopathy and sperm nuclear stability in vitro (Bedford et al., 1973a; Evenson et al., 1980). All previous studies of human sperm nuclear stability have been made with semen from infertile patients or from men of unknown fertility. In this communication we report studies of the normal biology of these phenomena, which were carried out with semen from men with recent, proven fertility.

\section{Materials and Methods}

\section{Semen specimens}

Semen specimens were obtained from 15 fertile men who were donors for therapeutic artificial insemination or men with proven paternity within 6 months from the time of specimen collection. All specimens were collected by masturbation into wide-mouth polypropylene specimen containers and were allowed to liquefy at room temperature for $30-45 \mathrm{~min}$. Five semen samples were collected from 4 of these donors at approximately monthly intervals. Semen specimens from bulls (2), rabbits (2) and dogs (2) were collected with an artificial vagina and were used in the experiments within $90 \mathrm{~min}$ of collection.

\section{Assessment of semen quality}

Human ejaculates were assessed for volume, sperm concentration, sperm motility and sperm morphology. Ejaculate volume was measured with a glass pipette and sperm concentration was determined by duplicate haemocytometer counts. Sperm motility was assessed using videomicrography as described by Katz \& Overstreet (1981). In each ejaculate, percentage motility and mean swimming speed were determined from a sample of 50 cells. The morphology of 50 immobilized spermatozoa per sample was also assessed by videomicrography (Overstreet, Price, Blazak, Lewis \& Katz, 1981).

\section{Assessment of the nuclear stability of spermatozoa}

A $40 \mu \mathrm{l}$ aliquot of the sperm suspension was mixed with $0.36 \mathrm{ml}$ of a $1 \%(\mathrm{w} / \mathrm{v})$ sodium dodecyl sulphate (SDS) solution prepared in 0.05 M-sodium borate buffer (pH 9.0) (Bedford $e t$ al., 1973a). SDS is an anionic detergent which disrupts non-covalent bonds and has been shown to remove the plasma membrane and acrosomal membranes from mammalian spermatozoa (Calvin \& Bedford, 1971). After $1 \mathrm{~h}, 5 \mu \mathrm{l}$ of the SDS-treated sperm suspension were examined with phase-contrast microscopy using $\times 20$ and $\times 40$ objectives. The nuclei of 100 consecutive spermatozoa per sample were classified as stable (S), moderately decondensed (M) or grossly decondensed (G) (see Bedford et al., 1973a; Kvist \& Eliasson, 1980; Plate 1). 
PLATE 1

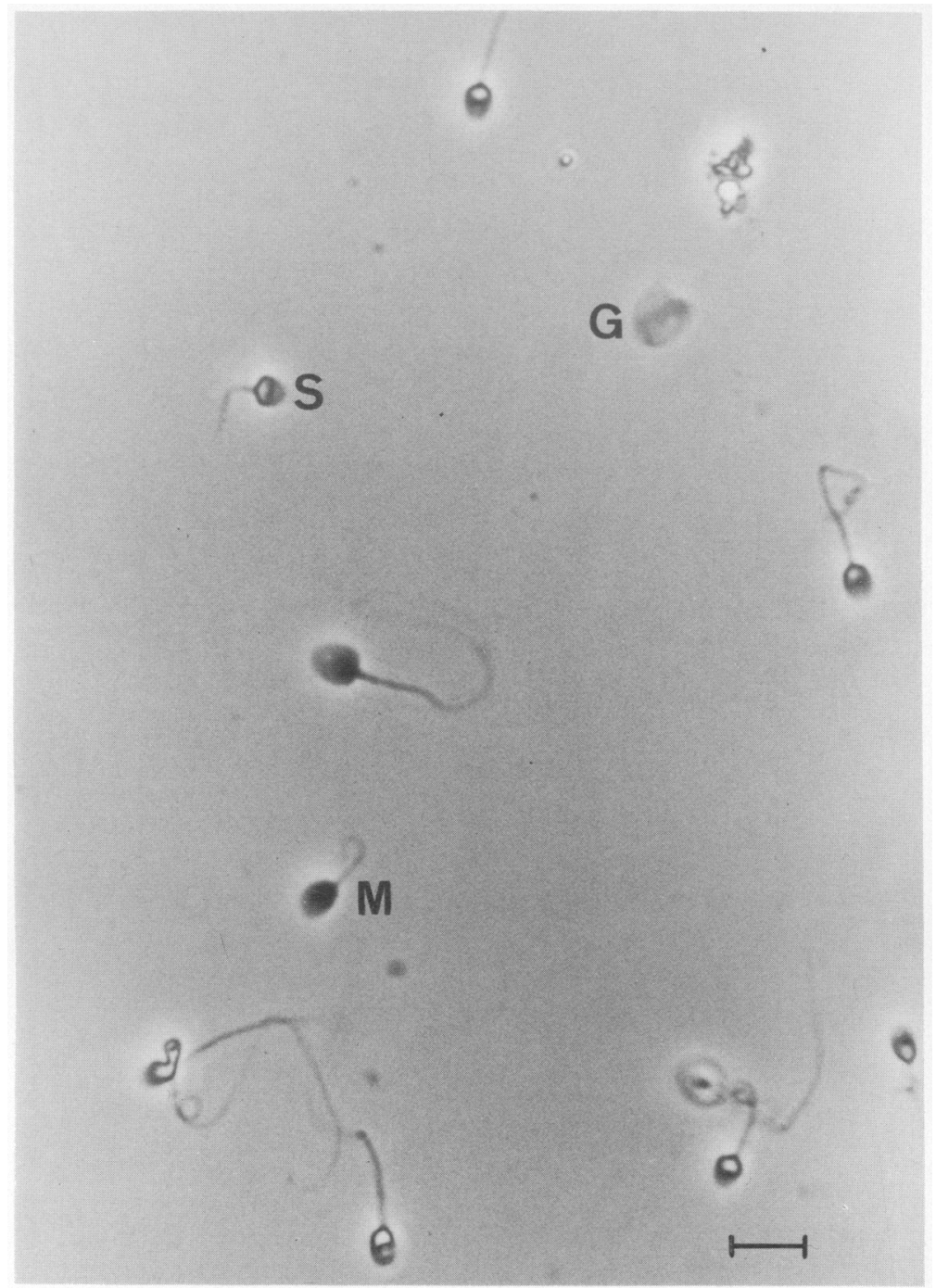

Phase-contrast photomicrographs of ejaculated human spermatozoa after treatment in $1 \%$ sodium dodecyl sulphate for $1 \mathrm{~h}$. The nuclei were classified as follows: Stable $(S)$ - the nucleus appeared highly refractile and showed no visible signs of chromatin decondensation; moderately decondensed $(M)$ - the nucleus appeared darkened and somewhat larger in size reflecting a moderate degree of chromatin decondensation; grossly decondensed $(G)$ - the nucleus appeared almost transparent with a colour approaching that of the background illumination and had markedly increased in size, reflecting an extensive degree of chromatin decondensation. Bar equals $10 \mu \mathrm{m}$. 


\section{Experiments}

Experiment 1. To determine the effects of washing on sperm nuclear stability, $0.5 \mathrm{ml}$ semen was diluted to a total volume of $5 \mathrm{ml}$ with a modified version of the medium of Biggers, Whitten \& Whittingham (1971) as modified by Yanagimachi et al. (1979). The medium contained $3 \mathrm{mg}$ bovine serum albumin (BSA, Sigma, Fraction $\mathrm{V}) / \mathrm{ml}$ and is hereafter referred to as BWW medium. The resulting sperm suspensions were centrifuged for $6 \mathrm{~min}$ at approximately $500 \mathrm{~g}$, the supernatant was removed, and the pellet was re-suspended in $0.5 \mathrm{ml} \mathrm{BWW}$ medium. The spermatozoa studied at this stage were considered to be washed once (i.e. $\times 1$ wash). Such washing procedures are typical of those employed when human spermatozoa are prepared for in-vitro interaction with human or zona-free hamster ova (e.g. Yanagimachi et al., 1979). For the second and all additional washes, the sperm suspension was diluted to a total volume of $5 \mathrm{ml}$ with BWW medium, re-centrifuged and re-suspended in BWW medium as previously described. A total of 5 washes was conducted. Before washing, and after the first, third and fifth washes, the percentages of motile cells were determined by videomicrography and nuclear stability was assessed after $1 \mathrm{~h}$ of treatment in SDS. Semen samples from 10 fertile men were studied in this experiment.

Experiment 2. To assess the effects of the supernatant fluid on the degree of nuclear chromatin decondensation of washed spermatozoa, the sperm pellet resulting after the fifth centrifugation was divided into three aliquots. These were resuspended in $0.2 \mathrm{ml}$ of the supernatant from the first wash, the supernatant of the third wash, or fresh BWW medium. After $10 \mathrm{~min}$ the cells were added to the SDS solution and nuclear stability was assessed $1 \mathrm{~h}$ later. The experiment was replicated 4 times with semen from 4 different fertile donors.

Experiment 3. To assess the effects of zinc and copper on the nuclear decondensation of washed human sperm, $40 \mu \mathrm{l}$ aliquots of $\mathrm{ZnCl}_{2}$ (prepared in $1 \mathrm{mM}-\mathrm{HCl}$ plus $137 \mathrm{~mm}-\mathrm{NaCl}$ ) or $\mathrm{CuCl}_{2}$ (prepared in the same diluent) were added to $\times 5$ washed sperm suspensions $(0.36 \mathrm{ml})$ to a final concentration of $1 \mathrm{~mm}$-zinc or $1 \mathrm{mM}$-copper. Copper is believed to promote the oxidation of free sulphydryl groups (-SH) to disulphide bridges (-S-S-), whereas zinc binds to free sulphydryl groups but does not promote their oxidation to disulphides (see Calvin, Yu \& Bedford, 1973). The control suspensions in this experiment were treated with the $\mathrm{HCl}$-sodium chloride diluent. Each sperm suspension was incubated at $37^{\circ} \mathrm{C}$ in air for $1 \mathrm{~h}$ and nuclear decondensation in SDS was assessed as previously described. The experiment was replicated 5 times with semen from 5 different fertile donors.

\section{Statistical analysis}

Differences in the mean percentages of cells with stable (S) nuclei after treatment in SDS were tested with the Student's $t$ test. In some cases, $\chi^{2}$ tests were used to examine the significance of differences between results obtained with spermatozoa from different donors.

\section{Results}

\section{The stability of sperm nuclei in ejaculates of fertile men}

The characteristics of semen quality, including semen volume, sperm concentration, motility, and morphology, for the fertile men in this study are given in Table 1 . These values were within the range of values considered normal for a fertile human male (Eliasson, 1975). The majority of sperm nuclei remained stable after treatment with SDS, but 2 of the men had $<70 \%$ stable sperm nuclei after the SDS treatment. There was no apparent relationship between the percentage of cells with stable nuclei and any classical semen characteristic (Table 1).

The percentage of ejaculated spermatozoa with SDS-stable nuclei was unchanged in repeated ejaculates collected from 4 donors at intervals of approximately 30 days (Table 2 ). This 
Table 1. Semen characteristics and nuclear stability after sodium dodecyl sulphate (SDS) treatment of spermatozoa from 15 fertile men

\begin{tabular}{|c|c|c|c|c|c|c|c|c|}
\hline \multirow[b]{2}{*}{ Donor } & \multirow[b]{2}{*}{$\begin{array}{l}\text { Ejaculate } \\
\text { vol. (ml) }\end{array}$} & \multirow[b]{2}{*}{$\begin{array}{c}\text { Sperm } \\
\text { conc. } \\
\left(\times 10^{6} \text { cells } / \mathrm{ml}\right)\end{array}$} & \multicolumn{2}{|c|}{ Sperm motility } & \multirow{2}{*}{$\begin{array}{c}\begin{array}{c}\text { Sperm } \\
\text { morphology }\end{array} \\
\begin{array}{c}\% \text { of } \\
\text { oval cells* }\end{array}\end{array}$} & \multicolumn{3}{|c|}{ Sperm nuclear stability $\dagger$} \\
\hline & & & $\begin{array}{l}\% \text { of } \\
\text { motile } \\
\text { cells* }\end{array}$ & $\begin{array}{c}\text { Mean } \\
\text { swimming } \\
\text { speed* } \\
(\mu \mathrm{m} / \mathrm{sec})\end{array}$ & & $\%$ Stable & $\begin{array}{c}\% \\
\text { Moderately } \\
\text { decondensed }\end{array}$ & $\begin{array}{c}\% \\
\text { Grossly } \\
\text { decondensed }\end{array}$ \\
\hline A & 3.7 & 96 & 76 & 39 & 70 & 98 & 2 & 0 \\
\hline B & 4.0 & 44 & 62 & 44 & 66 & 97 & 3 & 0 \\
\hline C & 2.8 & 59 & 80 & 46 & 52 & 97 & 3 & 0 \\
\hline D & 4.4 & 128 & 78 & 29 & 62 & 95 & 5 & 0 \\
\hline $\mathrm{E}$ & 2.9 & 111 & 62 & 37 & 68 & 95 & 4 & 1 \\
\hline $\mathbf{F}$ & 3.0 & 62 & 58 & 51 & 62 & 94 & 5 & 1 \\
\hline G & $3 \cdot 1$ & 38 & 82 & 32 & 58 & 92 & 7 & 1 \\
\hline $\mathbf{H}$ & 5.9 & 92 & 70 & 48 & 78 & 90 & 9 & 1 \\
\hline I & $2 \cdot 0$ & 139 & 74 & 46 & 64 & 90 & 7 & 3 \\
\hline $\mathrm{J}$ & 5.9 & 101 & 70 & 35 & 68 & 90 & 7 & 3 \\
\hline $\mathrm{K}$ & $3 \cdot 3$ & 147 & 64 & 44 & 76 & 89 & 9 & 2 \\
\hline L & 3.7 & 97 & 80 & 43 & 54 & 86 & 10 & 4 \\
\hline $\mathbf{M}$ & 3.7 & 78 & 54 & 60 & 72 & 85 & 11 & 4 \\
\hline $\mathbf{N}$ & $4 \cdot 1$ & 32 & 78 & 28 & 70 & 67 & 27 & 6 \\
\hline $\mathbf{0}$ & $4 \cdot 0$ & 49 & 66 & 41 & 80 & 65 & 28 & 7 \\
\hline $\begin{array}{c}\text { Mean } \pm \\
\text { s.e.m. }\end{array}$ & $3 \cdot 8 \pm 0.3$ & $85 \pm 10$ & $70 \pm 2$ & $41 \pm 2$ & $67 \pm 2$ & $89 \pm 3$ & $9 \pm 3$ & $2 \pm 1$ \\
\hline
\end{tabular}

- Based on 50 cells/ejaculate.

$\dagger$ See Plate 1.

Table 2. Nuclear stability after SDS treatment of spermatozoa in repeated ejaculates from 4 fertile men

\begin{tabular}{ccccc}
\hline & \multicolumn{4}{c}{ Donor } \\
\cline { 2 - 5 } Ejaculate* $^{n}$ & $\mathrm{~A}$ & $\mathrm{~J}$ & $\mathrm{~L}$ & $\mathrm{~N}$ \\
\hline 1 & 98 & 90 & 86 & 67 \\
2 & 93 & 89 & 87 & 76 \\
3 & 88 & 87 & 88 & 60 \\
4 & 93 & 88 & 81 & 66 \\
5 & 92 & 91 & 84 & 62 \\
Mean \pm s.e.m. & $93 \pm 2$ & $89 \pm 1$ & $85 \pm 1$ & $66 \pm 3$ \\
\hline
\end{tabular}

* Ejaculates were examined at intervals of approximately 30 days.

Each value in the table is the percentage of cells with stable nuclei after treatment in SDS.

was true for donors with a high percentage of stable cells and for one donor with a significant proportion of unstable nuclei.

\section{Experiment 1: effects of washing on sperm nuclear stability}

The overall mean percentage of cells with stable nuclei decreased $(P<0.01)$ after 5 cycles of washing in BWW medium (Table 3). The overall mean ( \pm s.e.m.) percentage of motile 
Table 3. Nuclear stability of human spermatozoa from fertile men before and after washing with BWW medium

\begin{tabular}{|c|c|c|c|c|c|c|c|c|c|c|c|c|}
\hline \multirow[b]{2}{*}{ Donor } & \multicolumn{3}{|c|}{ Wash 0} & \multicolumn{3}{|c|}{ Wash 1} & \multicolumn{3}{|c|}{ Wash 3} & \multicolumn{3}{|c|}{ Wash 5} \\
\hline & $\% \mathrm{~S}$ & $\% M$ & $\% G$ & $\% \mathrm{~S}$ & $\% \mathrm{M}$ & $\% G$ & $\% \mathrm{~S}$ & $\% \mathrm{M}$ & $\% G$ & $\% S$ & $\% \mathrm{M}$ & $\% G$ \\
\hline A & 86 & 11 & 3 & 68 & 27 & 5 & 54 & 25 & 21 & 56 & 28 & 16 \\
\hline C & 90 & 9 & 1 & 84 & 13 & 3 & 54 & 32 & 14 & 55 & 26 & 19 \\
\hline D & 95 & 5 & 0 & 90 & 6 & 4 & 73 & 13 & 14 & 66 & 12 & 22 \\
\hline E & 92 & 8 & 0 & 82 & 16 & 2 & 68 & 30 & 2 & 54 & 27 & 19 \\
\hline G & 95 & 4 & 1 & 81 & 17 & 2 & 70 & 22 & 8 & 62 & 21 & 17 \\
\hline $\mathrm{J}$ & 88 & 10 & 2 & 80 & 18 & 2 & 64 & 28 & 8 & 56 & 28 & 16 \\
\hline $\mathrm{K}$ & 90 & 8 & 2 & 67 & 17 & 16 & 33 & 44 & 23 & 39 & 38 & 23 \\
\hline L & 80 & 17 & 3 & 72 & 25 & 3 & 29 & 50 & 21 & 25 & 26 & 49 \\
\hline $\mathrm{N}$ & 66 & 26 & 8 & 35 & 44 & 21 & 23 & 26 & 51 & 12 & 32 & 56 \\
\hline 0 & 65 & 28 & 7 & 40 & 46 & 14 & 27 & 43 & 30 & 35 & 38 & 23 \\
\hline Mean & 85 & 13 & 2 & 70 & 23 & 7 & 49 & 31 & 20 & 46 & 28 & 26 \\
\hline s.e.m. & 3 & 3 & 1 & 6 & 4 & 2 & 6 & 4 & 5 & 6 & 3 & 5 \\
\hline
\end{tabular}

$\% \mathrm{~S}$, percentage of cells with stable nuclei; $\% \mathrm{M}$, percentage of cells with nuclei exhibiting a moderate degree of chromatin decondensation; \%G, percentage of cells with nuclei exhibiting a gross degree of chromatin decondensation.

spermatozoa observed before washing $(73 \pm 5 \%)$ did not change significantly after 1,3 or 5 cycles of washing $(79 \pm 5 \%, 70 \pm 7 \%, 75 \pm 4 \%$, respectively). The significant change in nuclear stability occurred within 3 washes and there was no further difference after the third wash. Similar washing of ejaculated spermatozoa from dogs, rabbits, and bulls (2 replicates per species) did not affect the stability of the nuclei as assessed by SDS treatment. In all of the latter experiments, $100 \%$ of the sperm nuclei were stable after 5 washes.

There were significant differences amongst the donors in the response of the sperm nucleus to the washing procedures; e.g. a decline from 86 to $56 \%$ for donor A and 66 to $12 \%$ for Donor $\mathrm{N}$ after 5 washes. Although the percentage of cells with stable nuclei before washing was significantly correlated with the percentage of cells with stable nuclei after washing $(r=0.82$, $P<0.01$ ), a number of notable exceptions were apparent (e.g. Donors $\mathrm{C}$ and $\mathrm{K}$ in Table 3 ).

\section{Experiment 2: effects of the supernatant fluid on sperm nuclear stability after washing}

The results of the washing experiments could have been due to a change in the stability of the sperm nucleus as a result of washing, or to whole seminal plasma or seminal plasma contaminants in the supernatant of the early washes inhibiting the decondensation of sperm nuclear chromatin after the sperm membranes were removed with SDS. When sperm cells were washed five times and aliquots were resuspended in the supernatants of the first or third washes or in fresh BWW medium, the stability of sperm nuclei as assessed by SDS treatment declined after washing as expected, but the supernatant from the first $(\times 1)$ wash significantly inhibited nuclear decondensation in SDS (Text-fig. 1). A similar inhibition of nuclear decondensation was observed when whole seminal plasma, or the $\times 1$ supernatant, was transferred to the SDS solution before addition of $\times 5$ washed cells (data not shown).

\section{Experiment 3: effects of copper and zinc on sperm nuclear stability}

This experiment was to investigate the existence of free sulphydryl groups which were not already involved in disulphide bridges but could be so stabilized. A mean of $42 \pm 8 \%$ of washed cells remained stable in SDS when treated with saline alone (Text-fig. 2). Copper- and 


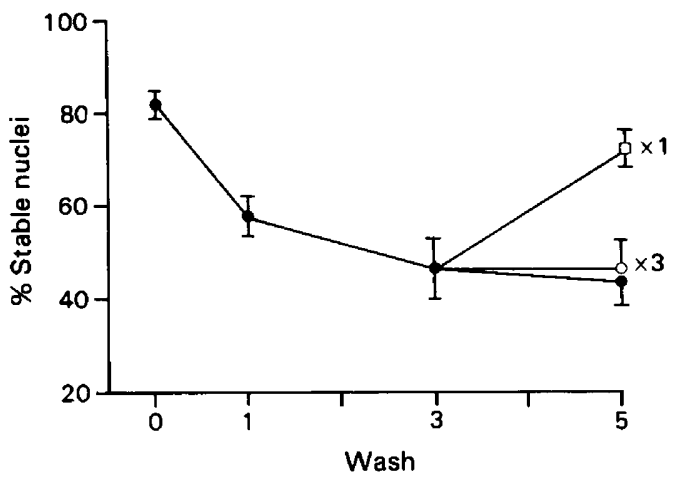

Text-fig. 1. The effects of the supernatant derived from washing ejaculated spermatozoa on the decondensation of sperm nuclear chromatin in SDS solution. Human semen samples $(n=4)$ were treated in SDS solution before (0 wash) and after 1, 3 and 5 washes in BWW medium (O). In addition to being resuspended in fresh BWW medium, aliquots of the cell suspensions after the fifth wash were also resuspended in the supernatants from the first $(\times 1)$ or third $(\times 3)$ washes. The percentages of cells with stable nuclei were determined after $1 \mathrm{~h}$ in SDS by phase-contrast microscopy.

zinc-treated cells, however, were significantly more resistant to nuclear chromatin decondensation in SDS (94 $\pm 2 \%$ and $88 \pm 4 \%$ stable cells respectively). After three washes, $40 \pm 9 \%$ of cells treated with saline alone remained stable in SDS whereas $91 \pm 2 \%$ of copper-treated cells remained stable. The percentage of stable zinc-treated cells declined linearly from $88 \pm 4 \%$ before washing to $54 \pm 9 \%$ after three washes.

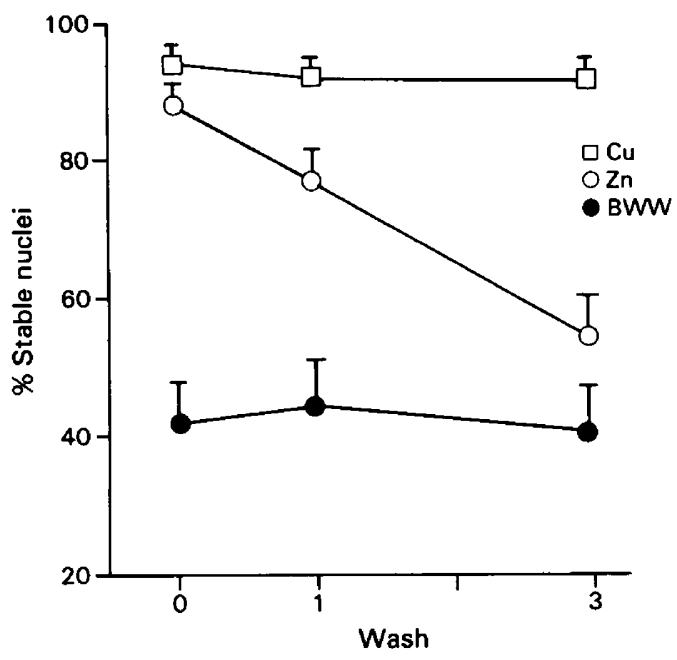

Text-fig. 2. The effects of copper and zinc on the decondensation of sperm nuclear chromatin in SDS solution. Human semen samples $(n=5)$ that had been washed 5 times were resuspended in BWW medium alone or in BWW medium containing $1 \mathrm{mM}-\mathrm{Cu}^{2+}$ or $1 \mathrm{mM}-\mathrm{Zn}^{2+}$. Aliquots of the resulting suspensions were then treated with SDS before ( 0 wash) or after 1 and 3 washes in BWW medium and the percentages of cells with stable nuclei were determined by phase-contrast microscopy. 


\section{Discussion}

The stabilization of human sperm nuclear chromatin has been attributed to the formation of disulphide bonds ( $-\mathrm{S}-\mathrm{S}-$ ) from thiol groups $(-\mathrm{SH})$ on adjacent nucleoprotein chains. The degree of disulphide bonding may depend in part on the relative proportions in the nucleus of the two types of protamine which differ in their cysteine content (Calvin, 1976). The formation of $-\mathrm{S}-\mathrm{S}-$ bonds is thought to occur during sperm transport through the epididymis (Bedford et al., 1973a), and the epididymal transit time as well as the epididymal environment may play a role in the regulation of the stabilization process (Calvin \& Bedford, 1971). The biological importance of sperm chromatin stability is not known, but a rigid sperm nucleus may be required for successful transport in the female reproductive tract and for penetration of the ovum vestments (Bedford \& Calvin, 1974). It has also been suggested that instability of the nucleus may be associated with male infertility and/or reproductive failure after fertilization (Bedford $e t$ al., 1973a). The degree of sperm nuclear chromatin condensation can be assessed directly by ultrastructural observations of the nucleus or indirectly by observing the response of the sperm nucleus to detergents such as SDS and/or reagents which cleave disulphide bonds such as dithiothreitol. Decondensation of the sperm nuclear chromatin in SDS alone, as reported in these studies, is considered an indication of inadequate $-\mathrm{S}-\mathrm{S}-$ stabilization of the nucleus (Bedford $e t$ at., 1973a).

The results of this study agree with previous observations of significant heterogeneity in the stability of sperm nuclei in human semen (Bedford et al., 1973a). However, the true proportion of unstable sperm nuclei in human ejaculates appears to be far larger than previous data would suggest. Our group of 15 fertile donors had a mean of $89 \%$ stable sperm cells and $87 \%$ of the men had $\geq 70 \%$ stable sperm nuclei in their ejaculates. The figures for a group of 44 men of unknown fertility were 84 and $89 \%$, respectively (Bedford et al., 1973a). Our experiments indicate that assessment of sperm nuclear stability in the presence of seminal plasma results in an artificially high assessment of stable sperm nuclei which is an artefact of the interaction of seminal plasma components (probably zinc) with the demembranated sperm head. When samples from some of our donors were re-examined after washing to remove the seminal plasma, only $46 \%$ of the nuclei remained stable in SDS and all of the 10 donors had fewer than $70 \%$ stable sperm nuclei after SDS treatment; $40 \%$ of the group had fewer than $50 \%$ stable cells.

Bedford et al. (1973a) suggested that decondensation of the sperm nucleus in SDS alone reflected an abnormality of testicular origin and that this might be associated with male infertility. The high degree of nuclear chromatin instability (up to $88 \%$ of cells undergoing nuclear decondensation) which we have seen in this group of fertile donors argues against both of these notions. It is our view that the heterogeneous response of human sperm nuclei to SDS is more likely to be due to the highly variable transit time of spermatozoa through the human epididymis (Rowley et al., 1970). Those spermatozoa which readily undergo nuclear decondensation in SDS may be structurally immature and their nuclei may be relatively poor in $-\mathbf{S}-\mathbf{S}-$ bonds. Our experiments with copper demonstrate that there are adequate sulphydryl groups available in these nuclei for oxidation to $-\mathbf{S}-\mathbf{S}-$ bonds. Those spermatozoa with unstable nuclei may be those which pass most rapidly through the epididymal tract.

Kvist \& Eliasson (1980) have reported significantly reduced zinc/fructose ratios in seminal plasma from ejaculates containing $<80 \%$ SDS-stable spermatozoa. Their experiments showed that the nuclear stability of such spermatozoa could be increased significantly by the addition of seminal plasma from men with a high (i.e. $290 \%$ ) frequency of SDS-stable spermatozoa. Because Kvist \& Eliasson (1980) were unable to detect a significant difference in the stability of sperm nuclei before and after a single wash, they concluded that a prostatic factor(s) was capable of directly associating with the nuclear chromatin after ejaculation, thus rendering the nucleus resistant to decondensation in SDS. Our experiments suggest a different interpretation. Such a prostatic factor (e.g. zinc) need not cross the sperm membrane to stabilize the sperm 
nucleus, because in this test system it may be present in the medium as a contaminant of the supernatant, and may thus stabilize the nucleus after SDS-induced demembranization.

A mechanism of thiol-disulphide exchange (e.g. Cecil, 1963) inherent to the sperm nucleus, could promote chromatin decondensation in a test system such as ours where no $-\mathrm{S}-\mathrm{S}-$ bond cleaving reagents are present. Such a mechanism has been postulated to act within the ooplasm at the time of fertilization (Kvist, 1980). It has been suggested that during sperm transport and capacitation in the female tract, zinc is lost from the sperm cells, freeing thiol groups to participate in thiol-disulphide exchange reactions after penetration of the ovum (Kvist, 1980). This hypothesis would thus suggest that at the time of fertilization sperm chromatin decondensation could proceed in the absence of a chemical contribution of the ovum, which others believe to be important (Thibault \& Gerard, 1970; Calvin \& Bedford, 1971; Usui \& Yanagimachi, 1976). We have found, however, that the nuclear stability of human spermatozoa, as assessed by treatment in SDS, does not change after capacitation in vitro (Blazak \& Overstreet, 1982). Furthermore, human spermatozoa can be readily capacitated in vitro in the presence of 125-500 $\mu \mathrm{M}$ zinc (Blazak \& Overstreet, 1982), which argues against the notion that loss of zinc from the spermatozoa is necessary for capacitation.

Since a significant proportion of ejaculated spermatozoa from fertile men are not extensively stabilized by $-\mathrm{S}-\mathrm{S}-$ bonds, the possibility must be considered that such spermatozoa could initiate abnormal embryonic development. There is no evidence to suggest that the presence of relatively unstable nuclear chromatin in a spermatozoon would necessarily imply that the genetic material is also abnormal. However, the nuclear DNA of mammalian spermatozoa appears to become more accessible to chemical agents (e.g. actinomycin D) after treatment of the cells with compounds, such as DTT, which reductively cleave disulphide bonds (Calvin \& Bedford, 1974; Incharoensakdi \& Panyim, 1981). If human spermatozoa contain a significant number of uncommitted thiol groups, as our experiments suggest, they may be more sensitive to mutagenic chemicals than are other mammalian spermatozoa. The possibility of such a differential sensitivity to mutagens requires immediate scientific attention, because of its implications for our current practice of human reproductive and genetic risk assessment by extrapolation from effects in laboratory animals.

W.F.B. was the recipient of a NIH Reproductive Biology Training Grant Postdoctoral Fellowship Award. J.W.O. acknowledges NIH Research Career Development Award No. HD 00224. This work was supported by NIH grant No. HD 15149. The technical assistance of $\mathrm{Mr}$ Raymond Tom is gratefully acknowledged.

\section{References}

Amann, R.P. \& Howards, S.S. (1980) Daily spermatozoal production and epididymal spermatozoal reserves of the human male. J. Urol. 124, $211-215$.

Bedford, J.M. (1975) Maturation, transport, and fate of spermatozoa in the epididymis. In Handbook of Physiology, Section 7, Endocrinology, Volume V, Male Reproductive System, Chapter 14, pp. 303-317. Eds D. W. Hamilton \& R. O. Greep. American Physiological Society, Washington, D.C.

Bedford, J.M. \& Calvin, H.I. (1974) The occurrence and possible functional significance of $-\mathrm{S}-\mathrm{S}-$ crosslinks in sperm heads, with particular reference to eutherian mammals. J. exp. Zool. 188, 137-156.

Bedford, J.M., Bent, M.J. \& Calvin, H. (1973a) Variations in the structural character and stability of the nuclear chromatin in morphologically normal human spermatozoa. J. Reprod. Fert. 33, 19-29.
Bedford, J.M., Calvin, H. \& Cooper, G.W. (1973b) The maturation of spermatozoa in the human epididymis. J. Reprod. Fert., Suppl. 18, 199-213.

Belonoschkin, B. (1942) Biologie der Spermatozoen in menschlichen Hoden und Nebenhoden. Arch. Gynaek. 174, 357-366.

Biggers, J.D., Whitten, W.K. \& Whittingham, D.G. (1971) The culture of mouse embryos in vitro. In Methods in Mammalian Embryology, p. 101 (Table 6-5). Ed. J. C. Daniel. Freeman, San Francisco.

Blazak, W.F. \& Overstreet, J.W. (1982) Zinc does not inhibit the capacitation of human spermatozoa in vitro. Gamete Res. 5, 153-160.

Calvin, H.I. (1976) Comparative analysis of the nuclear basic proteins in rat, human, guinea pig, mouse and rabbit spermatozoa. Biochem. Biophys. Acta 434, 377-389. 
Calvin, H. \& Bedford, J.M. (1971) Formation of disulphide bonds in the nucleus and accessory structures of mammalian spermatozoa during maturation in the epididymis. J. Reprod. Fert., Suppl. 13. 65-75.

Calvin, H.I. \& Bedford, J.M. (1974) Stimulation of actinomycin D-binding to eutherian sperm chromatin by reduction of disulphide bonds. J. Reprod. Fert. 36, 225-229.

Calvin, H.I., Yu, C.C. \& Bedford, J.M. (1973) Effects of epididymal maturation, zinc (II) and copper (II) on the reactive sulfhydryl content of structural elements in rat spermatozoa. Expl Cell Res. 81, 333-341.

Cecil, R. (1963) Intramolecular bonds in proteins. I. The role of sulfur in proteins. In The Proteins, 2nd edn, Vol. I, pp. 379-476. Ed. H. Nevrath. Academic Press, New York.

Eliasson, R. (1975) Analysis of semen. In Progress in Infertility, pp. 691-723. Eds S. J. Behrman \& R. W. Kistner. Little, Brown \& Co., Boston.

Evenson, D.P., Darzynkiewicz, Z. \& Melamed, M.R. (1980) Relation of mammalian sperm chromatin heterogeneity to fertility. Science, N.Y. 210, 11311133.

Hinrichsen, M.J. \& Blaquier, J.A. (1980) Evidence supporting the existence of sperm maturation in the human epididymis. J. Reprod. Fert. 60, 291-294.

Incharoensakdi, A. \& Panyim, S. (1981) In vitro decondensation of human sperm chromatin. Andrologia 13, 64-73.

Katz, D.F. \& Overstreet, J.W. (1981) Sperm motility assessment by videomicrography. Fert. Steril. 35, $188-193$.

Kvist, U. (1980) Importance of spermatozoal zinc as temporary inhibitor of sperm nuclear chromatin decondensation ability in man. Acta physiol. scand. 109, 79-84.
Kvist, U. \& Eliasson, R. (1980) Influence of seminal plasma on the chromatin stability of ejaculated human spermatozoa. Int. J. Androl. 3, 130-142.

McCosker, P.J. (1969) Abnormal spermatozoan chromatin in infertile bulls. J. Reprod. Fert. 18, 363-367.

Orgebin-Crist, M.C. (1969) Studies on the function of the epididymis. Biol. Reprod., Suppl. 1, 155-175.

Overstreet, J.W., Price, M.J., Blazak, W.F., Lewis, E.L. \& Katz, D.F. (1981) Simultaneous assessment of human sperm motility and morphology by videomicrography. J. Urol. 126, 357-360.

Rowley, M., Teshima, J.F. \& Heller, C.G. (1970) Duration of transit of spermatozoa through the human male ductular system. Fert. Steril. 21, $390-397$.

Saowaros, W. \& Panyim, S. (1979) The formation of disulfide bonds in human protamines during sperm maturation. Experientia 35, 191-192.

Thibault, C. \& Gerard, M. (1970) Facteur cytoplasmique nécessaire à la formation du pronucleus mâle dans l'ovocyte de lapine. C. r. hebd. Séanc. Acad. Sci. Paris D 270, 2025-2026.

Usui, N. \& Yanagimachi, R. (1976) Behavior of hamster sperm nuclei incorporated into eggs at various stages of maturation, fertilization and early development: the appearance and disappearance of factors involved in sperm chromatin decondensation in egg cytoplasm. J. Ultrastruct. Res. 57, 276288.

Yanagimachi, R., Lopata, A., Odom, C.B., Bronson, R.A., Mahi, C.A. \& Nicholson, G.L. (1979) Retention of the biologic characteristics of zona pellucida in highly concentrated salt solution: the use of salt-stored eggs for assessing the fertilizing capacity of spermatozoa. Fert. Steril. 31, 562-568.

Received 5 August 1981 